\title{
Asymptotic pointwise contractive type in modular function spaces
}

\author{
Farhan Golkarmanesh ${ }^{1 *}$ and Shahram Saeidi ${ }^{2}$
}

\author{
"Correspondence: \\ fgolkarmanesh@yahoo.com \\ 'Department of Mathematics, \\ Science and Research Branch, \\ Islamic Azad University, Tehran, Iran \\ Full list of author information is \\ available at the end of the article
}

\begin{abstract}
In this paper, we introduce asymptotic pointwise contractive type conditions in modular function spaces and present fixed point results for mappings under such conditions.
\end{abstract}

MSC: $47 \mathrm{H} 09 ; 47 \mathrm{H} 10 ; 54 \mathrm{H} 25$

Keywords: asymptotic pointwise $\rho$-contraction type; modular function space

\section{Introduction}

The notion of asymptotic pointwise contraction was introduced by Kirk [1]: Let $(M, d)$ be a metric space. A mapping $T: M \rightarrow M$ is called an asymptotic pointwise contraction if there exists a function $\alpha: M \rightarrow[0,1)$ such that for each integer $n \geq 1$,

$$
d\left(T^{n} x, T^{n} y\right) \leq \alpha_{n}(x) d(x, y) \quad \text { for each } x, y \in M,
$$

where $\alpha_{n} \rightarrow \alpha$ pointwise on $M$. Moreover, Kirk and Xu [2] proved that if $C$ is a weakly compact convex subset of a Banach space $E$ and $T: C \rightarrow C$ an asymptotic pointwise contraction, then $T$ has a unique fixed point $v \in C$, and for each $x \in C$, the sequence of Picard iterates $\left\{T^{n} x\right\}$ converges in norm to $v$.

Very recently, Saeidi [3] introduced the concept of (weak) asymptotic pointwise contraction type: Let $(M, d)$ be a metric space. A mapping $T: M \rightarrow M$ is said to be of asymptotic pointwise contraction type (resp. of weak asymptotic pointwise contraction type) if $T^{N}$ is continuous for some integer $N \geq 1$ and there exists a function $\alpha: M \rightarrow[0,1)$ such that for each $x$ in $M$,

$$
\begin{aligned}
& \limsup _{n \rightarrow \infty} \sup _{y \in M}\left\{d\left(T^{n} x, T^{n} y\right)-\alpha_{n}(x) d(x, y)\right\} \leq 0, \\
& \text { (resp. } \left.\liminf _{n \rightarrow \infty} \sup _{y \in M}\left\{d\left(T^{n} x, T^{n} y\right)-\alpha_{n}(x) d(x, y)\right\} \leq 0\right),
\end{aligned}
$$

where $\alpha_{n} \rightarrow \alpha$ pointwise on $M$.

It is easy to see that an asymptotic pointwise contraction is of asymptotic pointwise contraction type, but the converse is not true [3]. The following result was proved in [3].

Theorem 1.1 [3] Let $C$ be a nonempty weakly compact subset of a Banach space E, and let $T: C \rightarrow C$ be a mapping of weak asymptotic pointwise contraction type. Then $T$ has a

\section{Springer}

(c) 2013 Golkarmanesh and Saeidi; licensee Springer. This is an Open Access article distributed under the terms of the Creative Commons Attribution License (http://creativecommons.org/licenses/by/2.0), which permits unrestricted use, distribution, and reproduction in any medium, provided the original work is properly cited. 
unique fixed point $v \in C$ and, for each $x \in C$, the sequence of Picard iterates $\left\{T^{n} x\right\}$ converges in norm to $v$.

On the other hand, Khamsi and Kozlowski [4] studied the concept of asymptotic pointwise contractions in modular function spaces.

In this paper, motivated by Khamsi and Kozlowski $[4,5]$ and Saeidi [3], we study the notion of asymptotic pointwise contraction type in a modular function space. Moreover, we present fixed results which extend the earlier results in $[3,4]$.

\section{Preliminaries}

Let $\Omega$ be a nonempty set, and let $\Sigma$ be a nontrivial $\sigma$-algebra of subsets of $\Omega$. Let $\mathcal{P}$ be a $\delta$-ring of subsets of $\Omega$ such that $E \cap A \in \mathcal{P}$ for any $E \in \mathcal{P}$ and $A \in \Sigma$. Let us assume that there exists an increasing sequence of sets $K_{n} \in \mathcal{P}$ such that $\Omega=\bigcup K_{n}$. By $\xi$ we denote the linear space of all simple functions with supports from $\mathcal{P}$. By $\mathcal{M}_{\infty}$ we denote the space of all extended measurable function, i.e., all function $f: \Omega \rightarrow[-\infty,+\infty]$ such that there exists a sequence $\left\{g_{n}\right\} \in \xi,\left|g_{n}\right| \leq|f|$ and $g_{n}(\omega) \rightarrow f(\omega)$ for all $\omega \in \Omega$.

By $1_{A}$ we denote the characteristic function of the set $A$.

Definition 2.1 [6] Let $\rho: \mathcal{M}_{\infty} \rightarrow[0, \infty]$ be a nontrivial, convex and even function. We say that $\rho$ is a regular convex function pseudomodular if:

(a) $\rho(0)=0$;

(b) $\rho$ is monotone, i.e., $|f(\omega)| \leq|g(\omega)|$ for all $\omega \in \Omega$ implies $\rho(f) \leq \rho(g)$, where $f, g \in \mathcal{M}_{\infty}$;

(c) $\rho$ is orthogonally subadditive, i.e., $\rho\left(f 1_{A \cup B}\right) \leq \rho\left(f 1_{A}\right)+\rho\left(f 1_{B}\right)$ for any $A, B \in \Sigma$ such that $A \cap B \neq \emptyset, f \in \mathcal{M}_{\infty}$;

(d) $\rho$ has the Fatou property, i.e., $\left|f_{n}(\omega)\right| \uparrow|f(\omega)|$ for all $\omega \in \Omega$ implies $\rho\left(f_{n}\right) \uparrow \rho(f)$, where $f \in \mathcal{M}_{\infty}$;

(e) $\rho$ is order continuous in $\xi$, i.e., $g_{n} \in \xi$ and $\left|g_{n}(\omega)\right| \downarrow 0$ implies $\rho\left(g_{n}\right) \downarrow 0$.

Similarly as in the case of measure spaces, we say that a set $A \in \Sigma$ is $\rho$-null if $\rho\left(g 1_{A}\right)=0$ for every $g \in \xi$. We say that a property holds $\rho$-almost everywhere if the exceptional set is $\rho$-null. As usual we identify any pair of measurable sets whose symmetric difference is $\rho$-null as well as any pair of measurable functions differing only on a $\rho$-null set. With this in mind, we define

$$
\mathcal{M}(\Omega, \Sigma, \mathcal{P}, \rho)=\left\{f \in \mathcal{M}_{\infty}:|f(\omega)|<\infty \rho \text {-a.e. }\right\}
$$

where each $f \in \mathcal{M}(\Omega, \Sigma, \mathcal{P}, \rho)$ is actually an equivalence class of functions equal $\rho$-a.e. rather than an individual function. Where no confusion exists, we write $\mathcal{M}$ instead of $\mathcal{M}(\Omega, \Sigma, \mathcal{P}, \rho)$.

Definition 2.2 $[4,5]$ Let $\rho$ be a regular function pseudomodular;

(a) we say that $\rho$ is a regular convex function semimodular if $\rho(\alpha f)=0$ for every $\alpha>0$ implies $f=0 \rho$-a.e.;

(b) we say that $\rho$ is a regular convex function modular if $\rho(f)=0$ implies $f=0 \rho$-a.e.

The class of all nonzero regular convex function modulars on $\Omega$ is denoted by $\Re$. 
Definition 2.3 [7-9] Let $\rho$ be a convex function modular.

(a) A modular function space is the vector space $L_{\rho}(\Omega, \Sigma)$, or briefly $L_{\rho}$, defined by

$$
L_{\rho}=\{f \in \mathcal{M}: \rho(\lambda f) \rightarrow 0 \text { as } \lambda \rightarrow 0\} .
$$

(b) The following formula defines a norm in $L_{\rho}$ (frequently called Luxemburg norm):

$$
\|f\|_{\rho}=\inf \{\alpha>0 ; \rho(f / \alpha) \leq 1\} .
$$

In the following theorem, we recall some of the properties of modular function spaces that will be used later on in this paper.

Lemma 2.4 [7-9] Let $\rho \in \mathfrak{R}$. Defining $L_{\rho}^{0}=\left\{f \in L_{\rho} ; \rho(f, \cdot)\right.$ is order continuous $\}$ and $E_{\rho}=$ $\left\{f \in L_{\rho} ; \lambda f \in L_{\rho}^{0}\right.$ for every $\left.\lambda>0\right\}$, we have

(i) $L_{\rho} \supset L_{\rho}^{0} \supset E_{\rho}$;

(ii) $E_{\rho}$ has the Lebesgue property, i.e., $\rho\left(\alpha f, D_{k}\right) \rightarrow 0$, for $\alpha>0, f \in E_{\rho}$ and $D_{k} \downarrow \varnothing$;

(iii) $E_{\rho}$ is the closure of $\xi$ (in the sense of $\|\cdot\|_{\rho}$ ).

Definition 2.5 $[4,5]$ Let $\rho \in \Re$.

(a) We say that $\left\{f_{n}\right\}$ is $\rho$-convergent to $f$ and write $f_{n} \rightarrow f(\rho)$ if and only if $\rho\left(f_{n}-f\right) \rightarrow 0$.

(b) A sequence $\left\{f_{n}\right\}$ where $f_{n} \in L_{\rho}$ is called $\rho$-Cauchy if $\rho\left(f_{n}-f_{m}\right) \rightarrow 0$ as $m, n \rightarrow \infty$.

(c) A set $C \subset L_{\rho}$ is called $\rho$-closed if for any sequence $\left\{f_{n}\right\}$ in $C$, the convergence $f_{n} \rightarrow f(\rho)$ implies that $f$ belongs to $C$.

(d) A set $C \subset L_{\rho}$ is called $\rho$-bounded if $\sup \{\rho(f-g) ; f \in C, g \in C\}<\infty$.

(e) For a set $C \subset L_{\rho}$, the mapping $T: C \rightarrow C$ is called $\rho$-continuous if $f_{n} \rightarrow f(\rho)$, then $T\left(f_{n}\right) \rightarrow T(f)(\rho)$.

(f) A set $C \subset L_{\rho}$ is called $\rho$-a.e. closed if for any sequence $\left\{f_{n}\right\}$ in $C$ which $\rho$-a.e. converges to some $f$, then we must have $f \in C$.

(g) A set $C \subset L_{\rho}$ is called $\rho$-a.e. compact if for any sequence $\left\{f_{n}\right\}$ in $C$, there exists a subsequence $\left\{f_{n_{k}}\right\}$ which $\rho$-a.e. converges to some $f \in C$.

(h) Let $f \in L_{\rho}$ and $C \subset L_{\rho}$. The $\rho$-distance between $f$ and $C$ is defined as

$$
d_{\rho}(f, C)=\inf \{\rho(f-g) ; g \in C\} .
$$

Let us recall that $\rho$-convergence does not necessarily imply $\rho$-Cauchy condition. Also, $f_{n} \rightarrow f$ does not imply in general $\lambda f_{n} \rightarrow \lambda f, \lambda>1$.

Definition 2.6 [4] We say that $L_{\rho}$ has the property $(R)$ if and only if every nonincreasing sequence $\left\{C_{n}\right\}$ of nonempty, $\rho$-bounded, $\rho$-closed, convex subsets of $L_{\rho}$ has nonempty intersection.

Definition 2.7 [4] We say that the function modular $\rho$ is uniformly continuous if for every $\epsilon>0$ and $L>0$, there exists $\delta>0$ such that

$$
|\rho(g)-\rho(h+g)| \leq \epsilon \quad \text { if } \rho(h) \leq \delta \text { and } \rho(g) \leq L .
$$


Definition 2.8 [4] A function $\lambda: C \rightarrow[0, \infty]$, where $C \subset L_{\rho}$ is nonempty and $\rho$-closed, is called $\rho$-lower semicontinuous if for any $\alpha>0$, the set $C_{\alpha}=\{f \in C ; \lambda(f) \leq \alpha\}$ is $\rho$-closed.

It can be proved that $\rho$-lower semicontinuity is equivalent to the condition

$$
\lambda(f) \leq \liminf _{n \rightarrow \infty} \lambda\left(f_{n}\right) \quad \text { provided } f, f_{n} \in C \text { and } \rho\left(f-f_{n}\right) \rightarrow 0 .
$$

The following result plays an important role in the proof of the main results.

Lemma 2.9 [4] Assume that $\rho \in \mathfrak{R}$ has the property $(R)$. Let $C \subset L_{\rho}$ be nonempty, convex, $\rho$-closed and $\rho$-bounded. If $\varphi: C \rightarrow[0, \infty)$ is a $\rho$-lower semicontinuous convex function, then there exists $x_{0} \in C$ such that

$$
\varphi\left(x_{0}\right)=\inf \{\varphi(x) ; x \in C\} .
$$

Let us recall the notion of $\rho$-type.

Definition 2.10 [4] Let $C \subset L_{\rho}$ be convex and $\rho$-bounded. A function $\tau: C \rightarrow[0, \infty)$ is called a ( $\rho$ )-type (or shortly a type) if there exists a sequence $\left\{y_{m}\right\}$ of elements of $C$ such that for any $z \in C$, the following holds:

$$
\tau(z)=\limsup _{m \rightarrow \infty} \rho\left(y_{m}-z\right) .
$$

Lemma 2.11 [4] Let $\rho \in \mathfrak{R}$ be uniformly continuous. Let $C \subset L_{\rho}$ be nonempty, convex, $\rho$-closed and $\rho$-bounded. Then any $\rho$-type $\tau: C \rightarrow[0, \infty)$ is $\rho$-lower semicontinuous in $C$.

\section{Asymptotic pointwise contractive type conditions in modular function spaces}

Definition 3.1 [4] Let $\rho \in \mathfrak{R}$ and $C \subset L_{\rho}$ be non-empty and $\rho$-closed. A mapping $T$ : $C \rightarrow C$ is called an asymptotic pointwise mapping if there exists a sequence of mappings $\alpha_{n}: C \rightarrow[0,1]$ such that

$$
\rho\left(T^{n} f-T^{n} g\right) \leq \alpha_{n}(f) \rho(f-g) \quad \text { for any } f, g \in C .
$$

(a) If $\left\{\alpha_{n}\right\}$ converges pointwise to $\alpha: C \rightarrow[0,1)$, then $T$ is called asymptotic pointwise $\rho$-contraction.

(b) If $\lim \sup _{n \rightarrow \infty} \alpha_{n}(f) \leq 1$ for any $f \in C$, then $T$ is called asymptotic pointwise nonexpansive.

(c) If $\lim \sup _{n \rightarrow \infty} \alpha_{n}(f) \leq k$ for any $f \in C$ with $0<k<1$, then $T$ is called strongly asymptotic pointwise $\rho$-contraction.

Khamsi and Kozlowski proved the following results in modular function spaces.

Theorem 3.2 [4] Let $C \subset L_{\rho}$ be nonempty, $\rho$-closed and $\rho$-bounded. Let $T: C \rightarrow C$ be an asymptotic pointwise $\rho$-contraction. Then $T$ has at most one fixed point in $C$. Moreover, if $x_{0}$ is a fixed point of $T$, then the orbit $\left\{T^{n} x\right\}$ is $\rho$-convergent to $x_{0}$ for any $x \in C$. 
Theorem 3.3 [4] Let us assume that $\rho \in \mathfrak{R}$ is uniformly continuous and has the property $(R)$. Let $C \subset L_{\rho}$ be nonempty, convex, $\rho$-closed and $\rho$-bounded. Let $T: C \rightarrow C$ be an asymptotic pointwise $\rho$-contraction. Then $T$ has a unique fixed point $x_{0} \in C$. Moreover, the orbit $\left\{T^{n} x\right\}$ is $\rho$-convergent to $x_{0}$ for any $x \in C$.

Below, we introduce the notion of asymptotic pointwise $\rho$-contraction type in modular function spaces.

Definition 3.4 Let $C \subset L_{\rho}$ be nonempty, $\rho$-bounded and $\rho$-closed. A mapping $T: C \rightarrow C$ is said to be of asymptotic pointwise $\rho$-contraction type (resp. of weak asymptotic pointwise $\rho$-contraction type) if $T^{N}$ is $\rho$-continuous for some integer $N \geq 1$ and there exists a function $\alpha: C \rightarrow[0,1)$ such that, for each $x$ in $C$,

$$
\begin{aligned}
& \limsup _{n \rightarrow \infty} \sup _{y \in C}\left\{\rho\left(T^{n} x-T^{n} y\right)-\alpha_{n}(x) \rho(x-y)\right\} \leq 0, \\
& \left(\text { resp. } \liminf _{n \rightarrow \infty} \sup _{y \in C}\left\{\rho\left(T^{n} x-T^{n} y\right)-\alpha_{n}(x) \rho(x-y)\right\} \leq 0\right),
\end{aligned}
$$

where $\alpha_{n} \rightarrow \alpha$ pointwise on $M$.

Taking

$$
r_{n}(x)=\sup _{y \in M}\left\{\rho\left(T^{n} x-T^{n} y\right)-\alpha_{n}(x) \rho(x-y)\right\} \in \mathbb{R}^{+} \cup\{\infty\},
$$

it can be easily seen from (3.1) (resp. (3.2)) that

$$
\begin{aligned}
& \lim _{n \rightarrow \infty} r_{n}(x)=0, \\
& \left(\text { resp. } \liminf _{n \rightarrow \infty} r_{n}(x) \leq 0\right)
\end{aligned}
$$

for all $x \in M$, and

$$
\rho\left(T^{n} x-T^{n} y\right) \leq \alpha_{n}(x) \rho(x-y)+r_{n}(x) .
$$

We will obtain fixed point results for these mappings in modular function spaces.

First, it is worth mentioning that the $\rho$-limit of any $\rho$-convergent sequence in $L_{\rho}$ is unique. This fact follows from the following reasoning: Assume that $\rho\left(u_{n}-u\right) \rightarrow 0$ and $\rho\left(u_{n}-v\right) \rightarrow 0$. Then

$$
\rho\left(\frac{u-v}{2}\right) \leq \frac{1}{2} \rho\left(u-u_{n}\right)+\frac{1}{2} \rho\left(v-u_{n}\right) \rightarrow 0,
$$

which implies that $u=v$.

The following theorem is our main result.

Theorem 3.5 Let $\rho \in \Re$ be uniformly continuous and have the property $(R)$. Let $C \subset L_{\rho}$ be nonempty, convex, $\rho$-closed and $\rho$-bounded. Let $T: C \rightarrow C$ be a mapping of weak asymptotic pointwise $\rho$-contraction type. Then $T$ has a unique fixed point $v \in C$ and, for each $x \in C$, the sequence of Picard iterates $\left\{T^{n} x\right\}$ is $\rho$-convergent to $v$. 
Proof Fix an $x \in C$ and define a function $\tau$ by

$$
\tau(u)=\limsup _{n \rightarrow \infty} \rho\left(T^{n} x-u\right), \quad u \in C .
$$

By Lemma 2.11, $\tau$ is $\rho$-lower semicontinuous in $C$. By Lemma 2.9, then there exists $x_{0} \in C$ such that

$$
\tau\left(x_{0}\right)=\inf \{\tau(x) ; x \in C\} .
$$

Let us prove that $\tau\left(x_{0}\right)=0$. Indeed, for any $n, m \geq 1$, we have

$$
\begin{aligned}
\tau\left(T^{m} x_{0}\right) & =\limsup _{n \rightarrow \infty} \rho\left(T^{n} x-T^{m} x_{0}\right) \\
& =\limsup _{n \rightarrow \infty} \rho\left(T^{m+n} x-T^{m} x_{0}\right) \\
& =\limsup _{n \rightarrow \infty} \rho\left(T^{m}\left(T^{n} x\right)-T^{m} x_{0}\right) \\
& \leq \limsup _{n \rightarrow \infty} \alpha_{m}\left(x_{0}\right) \rho\left(T^{n} x-x_{0}\right)+r_{m}\left(x_{0}\right) \\
& =\alpha_{m}\left(x_{0}\right) \tau\left(x_{0}\right)+r_{m}\left(x_{0}\right),
\end{aligned}
$$

which implies

$$
\tau\left(x_{0}\right)=\inf \{\tau(x) ; x \in C\} \leq \tau\left(T^{m} x_{0}\right) \leq \alpha_{m}\left(x_{0}\right) \tau\left(x_{0}\right)+r_{m}\left(x_{0}\right) .
$$

Since $T$ is of weak asymptotic pointwise $\rho$-contraction type, by (3.4) we have $\liminf _{n \rightarrow \infty} r_{m}\left(x_{0}\right) \leq 0$. Thus, for a subsequence $\left\{r_{m_{k}}\left(x_{0}\right)\right\}$ of $\left\{r_{m}\left(x_{0}\right)\right\}$, we have

$$
\lim _{k \rightarrow \infty} r_{m_{k}}\left(x_{0}\right) \leq 0
$$

Now, by (3.6) and (3.7), we obtain

$$
\tau\left(x_{0}\right) \leq \liminf _{k \rightarrow \infty}\left[\alpha_{m_{k}}\left(x_{0}\right) \tau\left(x_{0}\right)+r_{m_{k}}\left(x_{0}\right)\right]=\alpha\left(x_{0}\right) \tau\left(x_{0}\right),
$$

which forces $\tau\left(x_{0}\right)=0$ as $\alpha\left(x_{0}\right)<1$. Hence $\rho\left(T^{n} x-x_{0}\right) \rightarrow 0$ as $n \rightarrow \infty$. From this and the continuity of $T^{N}$, for some $N \geq 1$, it follows that $\rho\left(T^{N+n} x-T^{N} x_{0}\right) \rightarrow 0$ as $n \rightarrow \infty$. Since the $\rho$-limit of any $\rho$-convergent sequence is unique, we must have $T^{N} x_{0}=x_{0}$, namely, $x_{0}$ is a fixed point of $T^{N}$. Now, repeating the above proof for $x_{0}$ instead of $x$, we deduce that $T^{n} x_{0}$ is $\rho$-convergent to a member $v$ of $C$; i.e., $\rho\left(T^{n} x_{0}-v\right) \rightarrow 0$. But $T^{k N} x_{0}=x_{0}$ for all $k \geq 1$. Hence, $v=x_{0}$ and then $T^{n} x_{0} \rightarrow x_{0}(\rho)$.

We show that $T x_{0}=x_{0}$; for this purpose, consider an arbitrary $\epsilon>0$. Then there exists a $k_{0}>0$ such that $\rho\left(T^{n} x_{0}-x_{0}\right)<\epsilon$ for all $n>k_{0}$. So, by choosing a natural number $k>k_{0} / N$, we obtain

$$
\rho\left(T x_{0}-x_{0}\right)=\rho\left(T\left(T^{k N} x_{0}\right)-x_{0}\right)=\rho\left(T^{k N+1} x_{0}-x_{0}\right)<\epsilon .
$$

Since the choice of $\epsilon>0$ is arbitrary and $\rho \in \mathfrak{R}$, we get $T x_{0}=x_{0}$. 
It is easy to verify that $T$ can have only one fixed point. Indeed, if $u, v \in C$ are fixed points of $T$, then by (3.5), we have

$$
\rho(u-v)=\rho\left(T^{n} u-T^{n} v\right) \leq \alpha_{n}(u) \rho(u-v)+r_{n}(u), \quad \forall n \geq 1 .
$$

Taking liminf in the above inequality, we obtain

$$
\rho(u-v) \leq \alpha(u) \rho(u-v) .
$$

Since $\alpha(u)<1$ and $\rho \in \Re$, we immediately get $u=v$.

Next, using the $\rho$-a.e. strong Opial property of the function modular, we prove a fixed point theorem which does not assume the uniform continuity of $\rho$.

Definition 3.6 $[4,10]$ We say that $L_{\rho}$ satisfies the $\rho$-a.e. strong Opial property (or shortly SO-property) if for every $\left\{f_{n}\right\} \in L_{\rho}$ which is $\rho$-a.e. convergent to zero such that there exists a $\beta>1$ for which

$$
\sup \left\{\rho\left(\beta f_{n}\right)\right\}<\infty
$$

the following equality holds for any $g \in L_{\rho}$ :

$$
\liminf _{n \rightarrow \infty}\left(f_{n}+g\right)=\liminf _{n \rightarrow \infty} \rho\left(f_{n}\right)+\rho(g) .
$$

Lemma 3.7 [4] Let $\rho \in \mathfrak{R}$. Assume that $L_{\rho}$ has the $\rho$-a.e. strong Opial property. Let $C \subset$ $E_{\rho}$ be a nonempty, $\rho$-a.e. compact subset such that there exists $\beta>1$ such that $\delta_{\rho}(\beta C)=$ $\sup \{\rho(\beta(x-y)) ; x, y \in C\}<\infty$. Let $D \subset C$ be a nonempty $\rho$-a.e. closed subset. For any $n \geq 1$, let $\lambda_{n}: D \rightarrow[0, \infty)$ be such that for any $y \in D$, there exists a sequence $\left\{y_{n}\right\} \subset C$ such that, for every $n \geq 1$, the following holds:

$$
\lambda_{n}(y)-\frac{1}{n} \leq \rho\left(y-y_{n}\right)
$$

and $\rho\left(x-y_{n}\right) \leq \lambda_{n}(x)$ for every $x \in D$ and $n \geq 1$. Let $\lambda(x)=\lim _{\sup _{n \rightarrow \infty}} \lambda_{n}(x)$ for any $x \in D$. Then there exists $x_{0} \in D$ at which $\lambda$ attains infimum, i.e.,

$$
\lambda\left(x_{0}\right)=\inf \{\lambda(x) ; x \in D\} .
$$

Theorem 3.8 Let $\rho \in \mathfrak{R}$. Assume that $L_{\rho}$ has the $\rho$-a.e. strong Opial property. Let $C \subset E_{\rho}$ be a nonempty $\rho$-a.e. compact convex subset such that $\delta_{\rho}(\beta C)=\sup \{\rho(\beta(x-y)) ; x, y \in C\}<$ $\infty$ for some $\beta>1$. Then any $T: C \rightarrow C$ of weak asymptotic pointwise $\rho$-contraction type has a unique fixed point $x_{0} \in C$. Moreover, the orbit $\left\{T^{n} x\right\}$ is $\rho$-convergent to $x_{0}$ for any $x \in C$.

Proof Fix an $x \in C$ and define a function $\tau$ by

$$
\tau(u)=\limsup _{n \rightarrow \infty} \rho\left(T^{n} x-u\right), \quad u \in C .
$$


By Lemma 3.7 applied with $\lambda(u)=\tau(u), D=C, \lambda_{n}(u)=\rho\left(T^{n} x-u\right)$, and with $y_{n}=T^{n} x$ chosen for all $u \in C$, there exists $x_{0} \in C$ such that

$$
\tau\left(x_{0}\right)=\inf \{\tau(x) ; x \in C\} .
$$

The rest of the proof is like the one used for Theorem 3.5.

\section{Competing interests}

The authors declare that they have no competing interests.

\section{Authors' contributions}

All the authors contributed equally. All authors read and approved the final manuscript.

\section{Author details}

'Department of Mathematics, Science and Research Branch, Islamic Azad University, Tehran, Iran. ${ }^{2}$ Department of Mathematics, University of Kurdistan, Sanandaj, 416, Iran.

\section{Acknowledgements}

The authors are grateful to the referees for their careful reading of the paper and several helpful comments.

Received: 9 September 2012 Accepted: 11 March 2013 Published: 17 April 2013

\section{References}

1. Kirk, WA: Asymptotic pointwise contraction. In: Plenary Lecture, the 8th International Conference on Fixed Point Theory and Its Applications. Chiang Mai University, Thailand, 16-22 July 2007 (2007)

2. Kirk, WA, Xu, H-K: Asymptotic pointwise contractions. Nonlinear Anal. 69, 4706-4712 (2008)

3. Saeidi, S: Mapping under asymptotic pointwise contractive type conditions. J. Nonlinear Convex Anal. (in press)

4. Khamsi, MA, Kozlowski, WM: On asymptotic pointwise contraction in modular function spaces. Nonlinear Anal. 73, 2957-2967 (2010)

5. Khamsi, MA, Kozlowski, WM: On asymptotic pointwise nonexpansive mappings in modular function spaces. J. Math. Anal. Appl. 380, 697-708 (2011)

6. Khamsi, MA: Fixed point theory in modular function spaces. In: Recent Advances on Metric Fixed Point Theorem, vol. 48, pp. 31-58. Universidad de Silva, Silva (1996)

7. Kozlowski, WM: Notes on modular function spaces I. Comment. Math. 28, 91-104 (1988)

8. Kozlowski, WM: Notes on modular function spaces II. Comment. Math. 28, 105-120 (1988)

9. Kozlowski, WM: Modular Function Spaces. Ser. Monogr. Textbooks Pure Appl. Math., vol. 122. Dekker, New York (1988)

10. Khamsi, MA: A convexity property in modular function spaces. Math. Jpn. 44, 269-279 (1996)

doi:10.1186/1687-1812-2013-101

Cite this article as: Golkarmanesh and Saeidi: Asymptotic pointwise contractive type in modular function spaces.

Fixed Point Theory and Applications 2013 2013:101.

\section{Submit your manuscript to a SpringerOpen ${ }^{\circ}$ journal and benefit from:}

- Convenient online submission

Rigorous peer review

- Immediate publication on acceptance

- Open access: articles freely available online

- High visibility within the field

- Retaining the copyright to your article 